\title{
PRIMER REGISTRO DEL CHUCAO SCELORCHILUS RUBECULA (KITTLITZ, 1830) (RHINOCRYPTIDAE) EN MAGALLANES.
}

\author{
FIRST RECORD OF SCELORCHILUS RUBECULA (KITTLITZ, \\ 1830) (RHINOCRYPTIDAE) IN MAGALLANES.
}

Jaime Cárcamoํㅡㄹ Juan M. Henríquez ${ }^{2,3}$ \& Alejandro Kusch ${ }^{3,4}$

La familia Rhinocryptidae es endémica de Sudamérica y está representada por cuatro géneros que incluyen ocho especies, de las cuales seis son propias del bosque templado lluvioso de la zona sur de Chile, encontrándose también algunas en Argentina (Fjeldsa \& Krabbe 1990, del Hoyo et al. 2003). En la región de Magallanes se han registrado sólo dos especies de esta familia: Pteroptochos tarnii (King) y Scytalopus magellanicus (Gmelin), siendo esta última la más común (Venegas \& Jory 1989, Venegas \& Sielfeld 1998). En la presente nota informamos la presencia del chucao, Scelorchilus rubecola (Kittlitz) al sur de su límite de distribución conocido hasta el momento, constituyéndose en una nueva especie para la región de Magallanes.

Durante los días 23 al 27 de noviembre del 2007 se realizó un levantamiento de información de diversidad de vertebrados terrestres en el sector de bahía Libertad (48 $88^{\prime}$ S, $74^{\circ} 04^{\prime} \mathrm{W}$ ), ubicado en la costa continental en el área de Angostura Inglesa, canal Messier. La vegetación del sitio corresponde a un bosque costero mixto del tipo siempreverde dominado por Nothofagus betuloides y Drymis winteri, rodeados por comunidades de turberas dominadas por Sphagnum magellanicum o por Donatia fasci-
cularis-Astelia pumila. Además, es posible observar densos matorrales costeros dominados por Tepualia stipularis y Gaultheria mucronata.

En una de las estaciones de escucha que se establecieron en el lugar se constató, mediante registro auditivo, la presencia de un ejemplar de chucao. Posteriormente se escucharon más cantos en las inmediaciones del sitio y se pudo registrar fotográficamente un ejemplar adulto (Fig. 1). En un área prospectada de 500 metros lineales sólo se detectó con seguridad la presencia de dos individuos. En otras localidades distribuidas en $57 \mathrm{~km}$ hacia el sur de bahía Libertad, tanto en isla Wellington como en el continente no se registró esta especie.

Los individuos observados ocupaban una quebrada protegida dominada por bosques mixtos, densos, sombríos y de gran humedad, dominando Nothofagus betuloides, el cual se observa como emergente, con ejemplares que superaban los 18 $\mathrm{m}$ de altura. En un segundo estrato se presentan especies arbóreas como Drimys winteri, Pilgerodendron uviferum, Embothrium coccineum, Lomatia ferruginea y Podocarpus nubigena. Sobre ellos se asocian especies trepadoras como Philesia magellanica, Mitraria coccinea y Lebethanthus myrsinites.

\footnotetext{
1 Laboratorio de Zoología, Instituto de la Patagonia, Universidad de Magallanes. Punta Arenas. jaime.carcamo@umag.cl

2 Laboratorio de Botánica, Instituto de la Patagonia, Universidad de Magallanes. Punta Arenas. jmarcos.henriquez@umag.cl

3 Centro de Ecología y Biodiversidad de la Patagonia Austral.

4 Consultora Biota Austral Ltd., biota.austral@gmail.com
} 


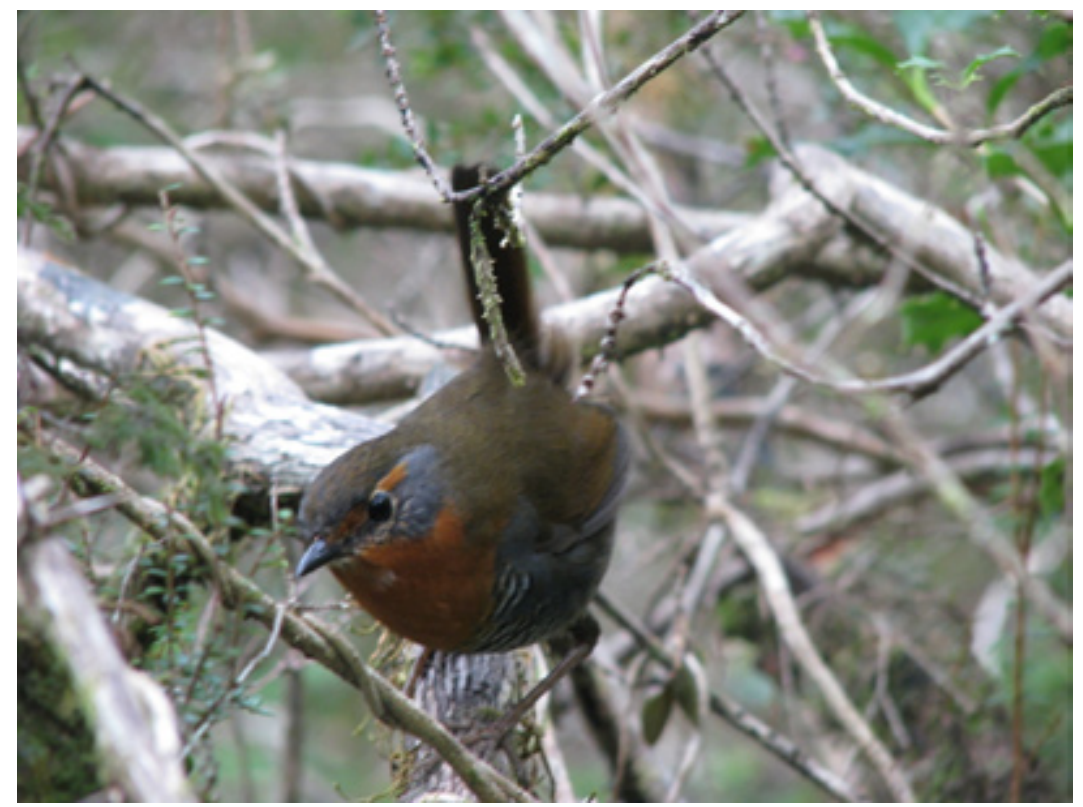

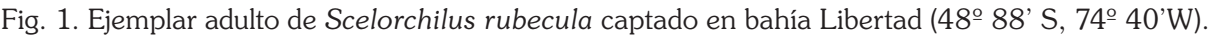

Un tercer estrato lo conforman especies arbustivas entre las que destacan Desfontania spinosa, Berberis ilicifolia, B. microphylla y Gaultheria mucronata.

Los fustes basales de las especies leñosas, comúnmente están cubiertos por helechos del género Hymenophyllum. Los suelos, a diferencia de las comunidades cercanas, no presentan capas continuas y profundas de musgos, siendo éstos interrumpidos por la presencia de rocas, especies herbáceas o helechos.

Históricamente la distribución del chucao en Chile se extiende desde el sur de Colchagua al golfo de Penas (Hellmayr 1932, Housse 1945, Goodall et al. 1951) y autores posteriores mantienen dicha distribución (e.g. Philippi 1964, Johnson 1967, Meyer de Schauensee 1970). Más recientemente, Venegas \& Sielfeld (1998) no lo mencionan para Magallanes; Jaramillo (2003) muestra un mapa donde amplía la distribución hasta el límite entre Aisén y Magallanes, no presenta datos de registros.

Un estudio específico en el área corresponde a Texera (1973) quien no encontró $S$. rubecula entre los $47^{\circ} 50^{\prime} \mathrm{S}$ y $52^{\circ} 45^{\prime} \mathrm{S}$ posiblemente porque el trabajo de campo se efectuó desde una embarcación y sólo consideró breves estadías en tierra. De manera similar, Imberti (2005), en censos de otoño, lo detectó de norte a sur sólo hasta bahía San Andrés (46 $\left.36^{\prime} \mathrm{S}, 70^{\circ} 30^{\prime} \mathrm{W}\right)$.
Existe un antecedente sin confirmación para el parque nacional Torres del Paine (Matus \& Barría 1999), y personal de CONAF residente en el sector de fiordo Témpano e isla Wellington mencionan que el chucao es común en el bosque, antecedente que tampoco había sido confirmado.

A partir de nuestros registros se expande la distribución sur del chucao hasta la región de Magallanes en aproximadamente 270 kilómetros considerando como límite anterior la localidad de puerto Barroso [ $\left(46^{\circ} 50\right.$ ' S, Port Otway en Wetmore 1923 y corrección de localidad en Paynter (1988)]. Aunque no se registró en otras localidades más hacia el sur creemos que es probable que existan otros sectores donde habite el chucao en la medida que existan bosques costeros con sotobosque denso y sin presentar discontinuidad o vacíos en el hábitat por disturbio antrópico (Wilson 2004). Un antecedente a considerar para futuros registros de esta especie en Magallanes es prospectar hábitats donde se cumplan dos requisitos mínimos, como son abundante sotobosque y que el tipo de suelo no sea turboso. Debido a que el chucao es un omnívoro de suelo donde busca semillas e insectos (Correa et al. 1990), es probable que los bosques de Nothofagus que tienen suelos inestables de turba no entreguen el recurso alimenticio en la cantidad o calidad necesaria. 


\section{AGRADECIMIENTOS}

Agradecemos al profesor Claudio Venegas por la revisión crítica al manuscrito. El estudio ha sido posible gracias al financiamiento de la Dirección de Investigación de la Universidad de Magallanes al programa 217-01. Se agradece a la Empresa Navimag y CONAF (LM Yepayek) por las facilidades en transporte.

\section{LITERATURA CITADA}

Correa, A., J. J. Armesto, R. P. Schlatter, R. Rozzi \& J. C. Torres-Mura 1990. La dieta del chucao (Scelorchilus rubecula), un Passeriforme terrícola endémico del bosque templado húmedo de Sudamérica austral. Revista Chilena de Historia Natural 63: 197 - 202.

Fjeldsa, J. \& N. Krabbe 1990. Birds of High Andes. Apollo Books, Svendborg, Denmark.

Goodall, J. D., A.W. Johnson \& R.A. Philippi 1951. Las aves de Chile, su conocimiento y sus costumbres. Vol. 2.

Hellmayr, C. E. 1932. The birds of Chile. Zool. Series, Pub. 308, Volume XIX, Field Mus. Nat. Hist., Chicago, Illinois.

Housse, R. 1945. Las Aves de Chile, su Vida y Costumbres. Ediciones de la Universidad de Chile.

Imberti, S. 2005. Distribución otoñal de aves marinas y terrestres en los canales chilenos. Anales Instituto Patagonia (Chile) 33:21-30.

Jaramillo, A. 2003. Birds of Chile. Helm Field Guides, Christopher Helm. London.
Johnson, A. W. 1967. The Birds of Chile and adjacent regions of Argentina, Bolivia and Peru. Volume II. Platt, Establecimientos Gráficos, Buenos Aires.

Matus, R. \& C. Barría 1999. Adiciones a la lista de aves del Parque Nacional Torres del Paine. Anales Instituto Patagonia, Serie Cs. Nat. (Chile) 27:105-113.

Meyer de Schauensee, R. 1982. A guide to the Birds of South America. Academy Natural Sciences of Philadelphia, Philadelphia, Pennsylvania.

Paynter, R. A. Jr. 1988. Ornithological Gazetteer of Chile. Museum of Comparative Zoology, Hardvard University, Cambridge, Massachussets.

Philippi, R.A. 1964. Catálogo de las aves de Chile con su distribución geográfica. Inv. Zool. Chilenas 11:1-179.

Texera, W. 1973. Distribución y diversidad de mamíferos y aves en la Provincia de Magallanes. II. Algunas notas ecológicas sobre los canales patagónicos. Anales Instituto Patagonia (Chile) 4:292-305.

Venegas, C. \& W. Sielfeld 1998. Catálogo de los Vertebrados de la Región de Magallanes y Antártica Chilena. Ediciones de la Universidad de Magallanes, Punta Arenas. 122 pp.

Wetmore, A. 1923. New subspecies of birds from Patagonia. University of California Publications in Zoology 21:333-337.

Willson, M. F. 2004. La pérdida de conectividad de hábitat impide la formación de parejas y la dispersión de Juveniles de Scelorchilus rubecula en la selva lluviosa de Chile. Condor 106: 
La Prensa Austral IMPRESOS.

Fono: 204012 / Fax: 247406 / Waldo Seguel 636

Punta Arenas - Chile 